\title{
Lack of Definite Association of Vitamin D Deficiency with Diabetic Neuropathy. Investigation in Greek and in Bangladeshi Patients
}

\author{
THOMAS ZAMBELIS ${ }^{1}$, GEORGE PAPADAKIS ${ }^{2}$, PANAGIOTIS KOKOTIS ${ }^{1}$, \\ VASSILIKI VILLIOTOU ${ }^{3}$, NIKOLAOS DOGKAS ${ }^{3}$ and NIKOLAOS KARANDREAS ${ }^{1}$ \\ ${ }^{1}$ Department of Neurology, National and Kapodistrian University of Athens, Aeginition Hospital, Athens, Greece; \\ ${ }^{2}$ Department of Endocrinology and Diabetes and \\ ${ }^{3}$ Biochemical Department, Metaxas Anticancer Hospital, Piraeus, Greece
}

\begin{abstract}
Aim: Determination of the 25(OH) vitamin D levels in Greek-born and in Bangladeshi immigrant patients in Greece with diabetes with and without polyneuropathy. Materials and Methods: The method for the detection and staging of polyneuropathy proposed by Dyck, 1988 was used. Results: A total of 111 Bangladeshi immigrants and 101 Greek diabetic patients took part in the study. Vitamin $D$ levels were significantly lower in Bangladeshi than in Greek diabetic patients, and were significantly lower in Greek patients with small-fiber neuropathy. In Bangladeshi patients, there was no statistically significant difference in the subgroup of patients with polyneuropathy in comparison to those without polyneuropathy. Conclusion: The association of vitamin D deficiency only with a small number of Greek patients with exclusively small-fiber neuropathy does not allow us to draw a definite conclusion on the role of vitamin $D$ in the pathogenesis of diabetic neuropathy.
\end{abstract}

Diabetic distal sensorimotor neuropathy (DSPN) is the most frequent type of polyneuropathy and the most frequent complication of diabetes affecting up to $50 \%$ of patients (1, 2). In several European studies, large-fiber neuropathy (LFN) and small-fiber neuropathy (SFN) seem to be less prevalent in Asian than in European patients with diabetes and this is attributed to their lower height, less smoking, and better skin microvascularization $(3,4)$. Vitamin D deficiency has also

This article is freely accessible online.

Correspondence to: Thomas Zambelis, Aeghinition Hospital, 74, Vass. Sofias Ave. 115-28, Athens, Greece. Tel/Fax: +30 2107250410, e-mail: thomzambelis@gmail.com

Key Words: Diabetic neuropathy, vitamin D, Greek, Bangladeshi. been recently implicated in the pathogenesis of diabetic neuropathy (DN) (5-7).

In a previous study, we showed that vitamin D levels were lower in Bangladeshi immigrants with diabetes in Greece compared to indigenous Greek patients with diabetes (8). In this study, we investigated the $25(\mathrm{OH})$ vitamin D level in patients with diabetes with and without polyneuropathy in both ethnic groups.

\section{Materials and Methods}

The study sample consisted of Greek and Bangladeshi patients recruited from the outpatient diabetic clinic of a general hospital and were matched as to age, sex and diabetes duration. This sample is part of that previously described (8). The following clinical and diabetes related factors were taken into consideration: Age, sex, type of diabetes, age at diabetes diagnosis, duration of diabetes, diabetes treatment, episodes of hypoglycemia, body mass index (BMI), glycated hemoglobin $(\mathrm{HbA} 1 \mathrm{c})$ and $25(\mathrm{OH})$ vitamin D levels. Patients treated for vitamin D deficiency, those taking vitamin D supplements and those treated with medicines known to affect bone metabolism were excluded from this study. The patients were recruited between January 2012 and December 2014, over a period of time that included all seasons. Serum $25(\mathrm{OH})$ vitamin D levels were measured using an assay kit from Roche Diagnostics $\mathrm{GmbH}$ (Mannheim, Germany) and the measurement was performed once, at the first appointment.

All patients gave their written consent and the study was approved by our local Ethics Committee (approval number 445/3011-2011).

The electrophysiological study for the detection and staging of polyneuropathy has been described elsewhere (9): The diagnosis of polyneuropathy required the combination of abnormal findings from the Neuropathy Symptoms Score (NSS), Neuropathy Disability Score (NDS), nerve conduction velocities (NCV) and Quantitative Sensory Tests (QST). The classification proposed by Dyck for patients with diabetes was used (10).

Student's $t$-test analysis was used to assess the statistical significance of the differences between groups. A value of $p<0.05$ was considered significant. 
in vivo $31: 259-262(2017)$

Table I. 25(OH) Vitamin D (VitD) levels in 101 Greek and in 111 Bangladeshi patients with diabetes according to polyneuropathy (PN).

\begin{tabular}{lcccc}
\hline & \multicolumn{2}{c}{ Greek } & & \multicolumn{2}{c}{ Bangladeshi } \\
\cline { 2 - 3 } Variable & VitD (ng/dl) & $p$-Value & VitD (ng/dl) & $p$-Value \\
\hline No PN & $23 \pm 12.4$ & & $12.4 \pm 5.9$ & $<0.01^{*}$ \\
PN & $20.25 \pm 12.9$ & NS** & $10.7 \pm 5.9$ & NS** \\
$\quad$ Exclusively LFN & $18.5 \pm 11$ & $<0.05^{* *}$ & $11.4 \pm 4.7$ & NS** \\
\hline
\end{tabular}

Data are the mean \pm SD. LFN: Large-fiber neuropathy, SFN: small-fiber neuropathy, NS: non significant. *Between ethnicities, **compared with the no polyneuropathy group.

\section{Results}

One hundred and eleven Bangladeshi immigrants (97 men and 14 women, mean age $=47.35$ years) and 101 consecutive Greek patients with diabetes (82 men and 19 women, mean age $=49.18$ years) were included in the study. The demographic characteristics of the two groups, the clinical and laboratory data of patients and diabetes complications and polyneuropathy have been described elsewhere (9).

Vitamin D levels were significantly lower in Bangladeshi than in Greek patients with diabetes without polyneuropathy $(12.4 \pm 5.9 v s .23 \pm 12.4 \mathrm{ng} / \mathrm{ml}, t$-test: $p<0.01)$. Table I shows $25(\mathrm{OH})$ vitamin D levels in groups of Greek and Bangladeshi patients with and without polyneuropathy. In Greek patients, the levels of vitamin D were significantly lower in those with SFN compared with those of the group without polyneuropathy (seven patients), but not in those with LFN (53 patients). In Bangladeshi patients, there was no statistically significant difference in the subgroup of patients with polyneuropathy in comparison with those without polyneuropathy ( $t$-test, $p>0.05$ ).

\section{Discussion}

In this study, vitamin D levels were lower in the group of Greek patients with diabetes and with SFN compared to the group without polyneuropathy, but not in that of the patients with LFN. There were, however, only 7 patients with SFN (6.9\%), while there were 53 patients with LFN (52.5\%).

In previous studies an association of low vitamin D levels with DN was found $(5-7,11-13)$. The method followed for the diagnosis of polyneuropathy was not uniform in these studies. Some authors used symptoms alone (5) or signs (11) of polyneuropathy, while others used nerve conduction studies only (7, 13), Shehab et al. used NSS, NDS and nerve conduction studies (6). We did not find any study to have used QST for the detection of SFN and separating LFN and SFN. Soderstrom et al. conceded that the incidence of DN had probably been underestimated in their sample (5). In our study the diagnosis of DN was achieved through a strict combination of NSS, NDS, NCV and QST. The guidelines for the diagnosis of DSPN of the American Association of Electrodiagnostic medicine (AAEM) state that the most accurate diagnosis of DSPN requires the combination of neuropathic signs, symptoms and abnormal electrophysiological findings, and that symptoms alone have poor diagnostic accuracy (14).

The pathogenesis of DN is not clearly understood. Vitamin $\mathrm{D}$ has been shown to have a neuroprotective effect (15) and to be implicated in the pathogenesis of diabetes mellitus (13, 16) and a low level of vitamin $D$ in patients with DN may simply indicate its insufficiency in patients with diabetes.

Levels of vitamin D differ in different seasons and our sample was recruited over a period of time that included all seasons. In Bangladeshis, we did not find any difference in $25(\mathrm{OH})$ vitamin D level in the subgroups of patients with DN. The levels of vitamin D in these patients were in any case very low. Bangladeshis mostly have type $\mathrm{V}$ skin (as defined on the Fitzpatrick scale) which protects against the sun, but also reduces absorption of ultraviolet B sunlight that is needed to produce vitamin $\mathrm{D}$ by the skin. Additionally, traditional Asian diets are not rich in vitamin D-containing foods. On the contrary, fair-skinned individuals synthesize more vitamin $\mathrm{D}$ when exposed to the same radiation regime (17). Bangladesh is one of the countries with the highest number of patients with diabetes mellitus (18), with a prevalence approximating $20 \%$ (3). It is also known that Asians develop diabetes at an earlier age than Europeans (19). The high incidence of DN in Bangladeshi might also in part be attributed to the low levels of $25(\mathrm{OH})$ vitamin $\mathrm{D}$.

We can postulate that vitamin $\mathrm{D}$ insufficiency is implicated in the pathogenesis and the high incidence of diabetes mellitus, but we cannot state this for DN.

In conclusion, the findings of vitamin D deficiency in a small number of Greek patients with exclusively SFN, but not in those with exclusively LFN, and the absence of such an association in Bangladeshis found in this study does not allow us to draw a definite conclusion on the role of vitamin $\mathrm{D}$ in the pathogenesis of DN. 


\section{References}

1 Tesfaye S, Boulton AJM, Dyck PJ, Freeman R, Horowitz M, Kempler P, Lauria G, Malik RA, Spallone V, Vinik A, Bernardi $\mathrm{L}$ and Valensi P: Diabetic neuropathies: Update on definitions, diagnostic criteria, estimation of severity, and treatments Diabetes Care 33: 2285-2293, 2010.

2 Pasnoor M, Dimachkie NM, Kluding P and Barohn RJ: Diabetic neuropathy part1: overview and symmetric phenotypes. Neurol Clin 31(2): 425-445, 2013.

3 Morkrid K, Ali L and Hussain A: Risk factors and prevalence of diabetic peripheral neuropathy: a study of type 2 diabetic outpatients in Bangladesh: Int J Diabetes Dev Ctries 30: 11-17, 2010.

4 Abbott CA, Chaturvedi N, Malik RA, Yates AP, Pemberton PW and Boulton AJM: Explanations for the lower rates of diabetic neuropathy in Indian Asians versus Europeans. Diabetes Care 33: 1325-1330, 2010.

5 Soderstrom LH, Johnson SP, Diaz VA and Mainous AG: Association between vitamin $\mathrm{D}$ and diabetic neuropathy in a nationally representative sample: results from 2001-2004 NHANES. Diabet Med 29(1): 50-55, 2012.

6 Shelab D, Al-Jarallah K, Mojiminiyi OA, Al Mohamedy H and Abdella NA: Does vitamin D deficiency play a role in peripheral neuropathy in type 2 diabetes? Diabet Med 29(1): 43-49, 2012.

7 Alamdari A, Mozafari R, Tafakhori A, Faghihi-Kashani S, Hafezi-Nejad N, Sheikhbahaei S, Naderi N, Ebadi M and Esteghamati A: An inverse association between vitamin D levels with the presence and severity of impaired nerve conduction velocity and large fiber peripheral neuropathy in diabetic subjects. Neurol Sci 36: 1121-1126, 2015.

8 Papadakis G, Zambelis T, Villiotou V, Dogkas N, Pappas A, Keramidas I, Kakava K, Michalopoulou M, Rosenberg T, Konstantopoulos K and Chatzipanagiotou S: Lower levels of vitamin D among Bangladeshi immigrants with diabetes in Greece compared to indigenous Greek patients with diabetes. In Vivo 29(5): 541-545, 2015.

9 Zambelis T, Papadakis G, Chatzipanagiotou S., Michalopoulou M, Kokotis P and Karandreas N: Diabetic neuropathy: comparison between Bangladeshi immigrants and Greek-born subjects. JPNS 19: 307-310, 2014.
10 Dyck PJ: Detection, characterization, and staging of polyneuropathy: Assessed in diabetics. Muscle Nerve 11: 21-32, 1988.

11 Skali S, Muller M, Pradines S, Halimi S and Wion-Barbot N: Vitamin D deficiency and peripheral diabetic neuropathy. Eur J Intern Med 23(2): e67-e68, 2012.

12 Celikbilek A, Gocmen AY, Tanik N, Borecki E, Adam M, Celikbilek M, Suher M and Delibas N: Decreased serum vitamin $\mathrm{D}$ levels are associated with diabetic peripheral neuropathy in a rural area of Turkey. Acta neurol Belg 115(1): 145-151, 2015.

13 Bajaj S, Singh RP, Dwivedi NC, Singh K, Gupta A and Mathur $\mathrm{M}$ : Vitamin D levels and microvascular complications in type 2 diabetes. Indian J Endocr Metabol 18(4): 537-541, 2014.

14 England JD, Grosneth GS, Franklin G, Miller RG, Asbury AK, Carter GT, Cohen JA, Fisher MA, Howard JF, Kinsella LJ, Latov N, Lewis RA, Low PA and Sumner AJ: AAEM practice topic in electrodiagnostic medicine. Distal symmetrical polyneuropathy: Definition for clinical research. Muscle Nerve 31: 113-123, 2005.

15 Riaz S, Malcangio M, Miller M and Tomlinson DR: A vitamin D3 derivate (CB1093) induces nerve growth factor and prevents neurotrophic deficits in streptozocin-diabetic rats. Diabetologia 42: 1308-1313, 1999.

16 Palomer X, Gonzalez-Clemente JM, Blanco-Vaca $\mathrm{F}$ and Mauricio D: Role of vitamin D. Diabetes: Obes Metab 10(3): 185-197, 2008.

17 Clemens TL, Adams JS, Henderson SL and Holick MF: Increased skin pigment reduces the capacity of skin to synthesise vitamin $\mathrm{D}_{3}$. Lancet 8263: 74-76, 1982.

18 Wild S, Roglic G and Green A: Global prevalence of diabetes. Estimates for the year 2000 and projection for 2030. Diabetes care 27: 1047-1053, 2004.

19 Chaturvedi N, Abbott CA, Whalley A, Widdows P, Leggetter SY and Boulton AJM: Risk of diabetes-related amputation in south Asians vs. Europeans in the UK. Diabet Med 19: 99-104, 2002.

Received December 18, 2016

Revised February 2, 2017

Accepted February 8, 2017 